\title{
A SOIL SEQUENCE \\ IN THE NATURAL AND RECLAIMED MARSHES \\ OF THE GUADALQUIVIR RIVER, SEVILLE (SPAIN)
}

\author{
F. Moreno, J. Martin \& J.L. Mudarra, Sevilla
}

\section{SUMMARY}

Five soil profiles from a saline zone in the province of Seville ("Marismas del Guadalquivir", SW. Spain) and one from an adjacent non-saline zone, have been analyzed in order to determine their physical and chemical properties and their classification.

All of these soils are recent and of fine texture. Hydraulic conductivity, except in top layers, as well as aeration porosity, is always low; non-available water holding capacity and linear extensibility, high. Differences are however found between those located near the river channel and those at the limit of Marismas in contact with vertisols, due to parent material, situation and use. Particularly noticeable is the progressive increment of their vertic character.

\section{INTRODUCTION}

Some $2000 \mathrm{~km}^{2}$ of Seville province (SW.Spain) is occupied by saline soils. They represent $14 \%$ of its total area.

These soils have developed after the infilling of an ancient lake (Lacus ligustinus), which was connected to the open sea during Roman times. According to the work of LEYVA (1976), the materials forming the Guadalquivir "Marisma" (marsh) are typical of sediments deposited at different levels. The oldest sediments have a thickness between 7 and $25 \mathrm{~m}$ and are mainly formed by very fine earths (silt and clay) in various proportions. These materials correspond to the old fluvial network of the Guadalquivir river, the morphology being typical of a river bed near to its mouth. Upon this section, lies another, partially discordant, thinner one $(20 \mathrm{~cm}$ to $5 \mathrm{~m}$ ), its lithology being similar to that of the underlying section in this lower part (argiolites and/or clayey limolites). This section occupies the largest part of the Marisma.

The recent sediments have been deposited on the lower parts, with altitudes between 0 and $2 \mathrm{~m}$, in depressions where there is stagnation of flood or run-off water from rainfall, enriched with salts as a consequence of evaporation.

The variety of soils found in this zone is important. In most cases, they contain predominantly clay of a non-expandible type (GONZALEZ GARCIA et al. 1956, MORENO et al. 1980) and the water surrounding the adsorbed cations is responsible for some of the soil properties, particularly the very high coefficient of linear extensibility.

The soils of this zone in the Marisma present an obvious handicap when used for agriculture, as their salt content is much too high for this purpose. To overcome this problem a series of reclamation projects were put into operation, the results of which can be found in GRANDE COVIAN (1967 and 1976). On the other hand certain areas have supported rice 
cultivation for more than 20 years in some cases. These facts pose the question about what may happen to these soil after several years of leaching by drainage or rice cultivation and what could be the physical, physico-chemical and chemical properties developed under these conditions due to either their situation within the Marisma or their cultivation. It is important also to consider the problems involved in the classification of these soils, particularly those devoted to rice cultivation as shown by KYUMA \& KAWAGUCHI (1967).

The aim of this work is to present and discuss the physical, physico-chemical and chemical properties derived from laboratory determinations on samples corresponding to profiles representative of various zones in the Guadalquivir Marisma and adjacent areas. The profiles studied belong to soils of various classes, from those still under natural conditions, to those under reclamation schemes or cultivated with rice. These different situations allow a comparison of their peculiar soil properties to be made. Finally, a classification of these soils, according to the Soil Taxonomy (U.S.D.A.), is proposed. Some aspects have already been dealt with in previous works by AYERS et al. (1960), GIRALDEZ \& CRUZ (1975), MARTIN et al. (1978), and MORENO et al. $(1978,1979)$.

\subsection{CLIMATE AND VEGETATION}

The climate is typically Mediterranean, with moderate humid winters and hot dry summers.

Mean temperature varies from $10.0^{\circ} \mathrm{C}$ (January) to $25.5^{\circ} \mathrm{C}$ (August). Maximum temperature, during summer period, frequently reaches $40^{\circ} \mathrm{C}$ and above.

Annual total rainfall varies from 350 to $1100 \mathrm{~mm}$, with an average of $550 \mathrm{~mm}$. Most of the precipitation falls from November to March. There is practically no rainfall during July and August.

The halophytic plant community of these saline soils belongs to the Order Thero-Salicornetalia within the Class Salicornificea. The most characteristic communities contain Chenopodiaceae, like: Beta vulgaris subsp. maritima, Arthrocnemum perenne, A. glaucum, Salicornia ramosissima, Suaeda vera, S. splendens, Salsola soda, together with other species, mainly: Spergularia salina, Melilotus indica, $M$. segetalis, Trifolium resupinatum, Frankenia laevis, Cressa cretica, Plantago coronopus var. maritima, Thrincia hispida, Sphenopus gouani, Aeloropus littoralis, Hordeum maritimum, Monerma cylindrica, Polypogon maritimus, Crypsis aculeata. Where flooding is persistent, Phragmiteteae, with Scirpus, Eleocharis, and Cyperus, are present.

\section{MATERIAL AND METHODS}

\subsection{SOILS}

The general area considered in this study is represented in fig. 1. Profiles have been chosen for each of the six subzones included in the scheme.

Profile 1 is representative of the most natural, undisturbed soil today. Profiles 1 and 4 represent the most saline parts in the area. Profile 6 is given as a contrasting example from a border situation and it is a typical vertisol. Profiles 2 and 3 are cultivated with wheat and rice, respectively; the latter under almost permanent flooding. Profiles 4 and 5 are situated in marginal zones; profile 4 without cultivation, profile 5 recently under wheat (see photo 1 ). 


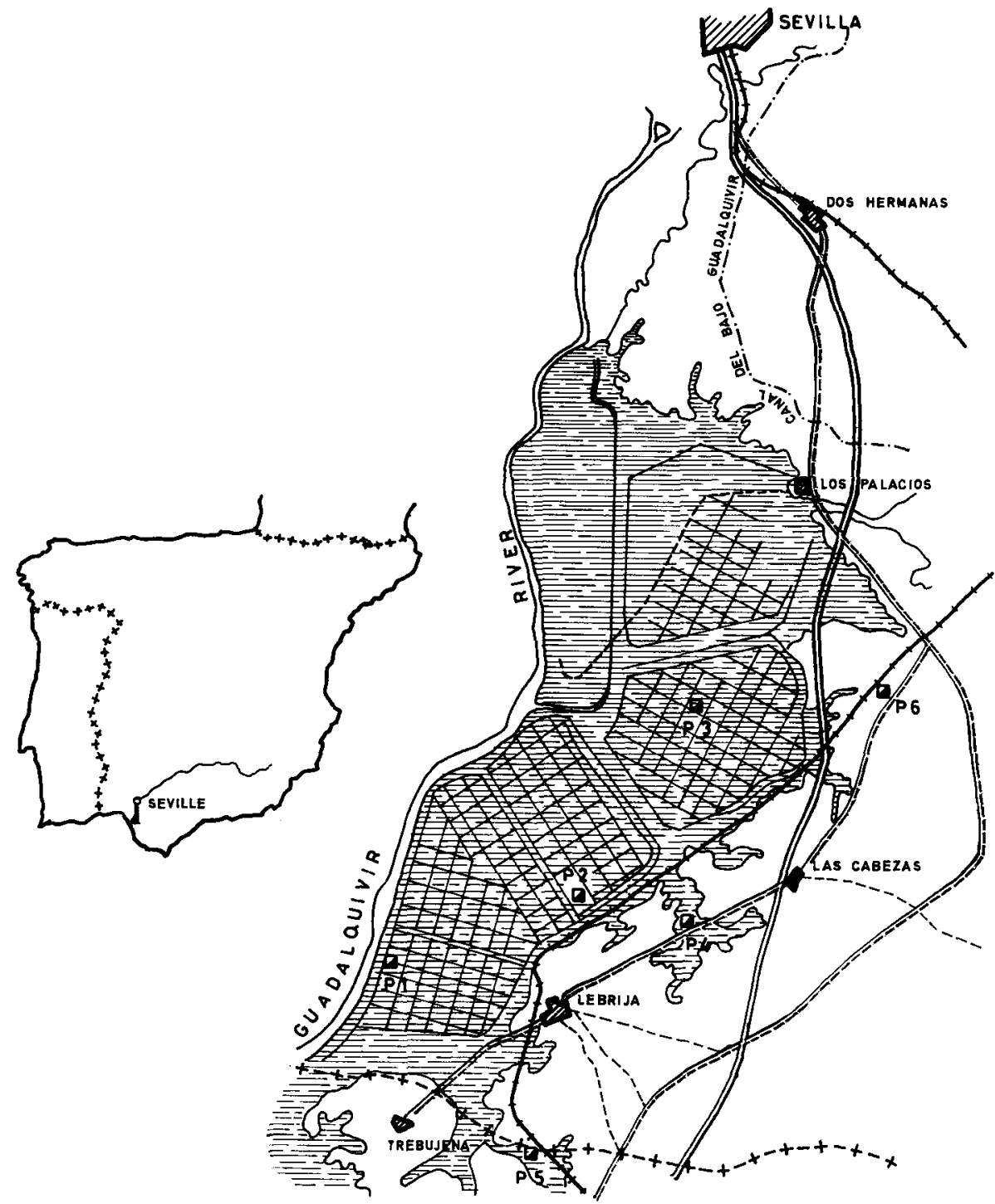

Fig. 1: Situation of profiles.

\subsection{SAMPLING}

In each profile samples have been taken at $20 \mathrm{~cm}$ intervals, except for the first layer, in which samples have been taken at $10 \mathrm{~cm}$ intervals. Stainless steel cylinders $(8.0 \mathrm{~cm}$ diameter by 4.0 and $2.0 \mathrm{~cm}$ high) with sharp edges have been employed. Samples, hermetically covered with plastic lids, were transported to the laboratory for analysis. 

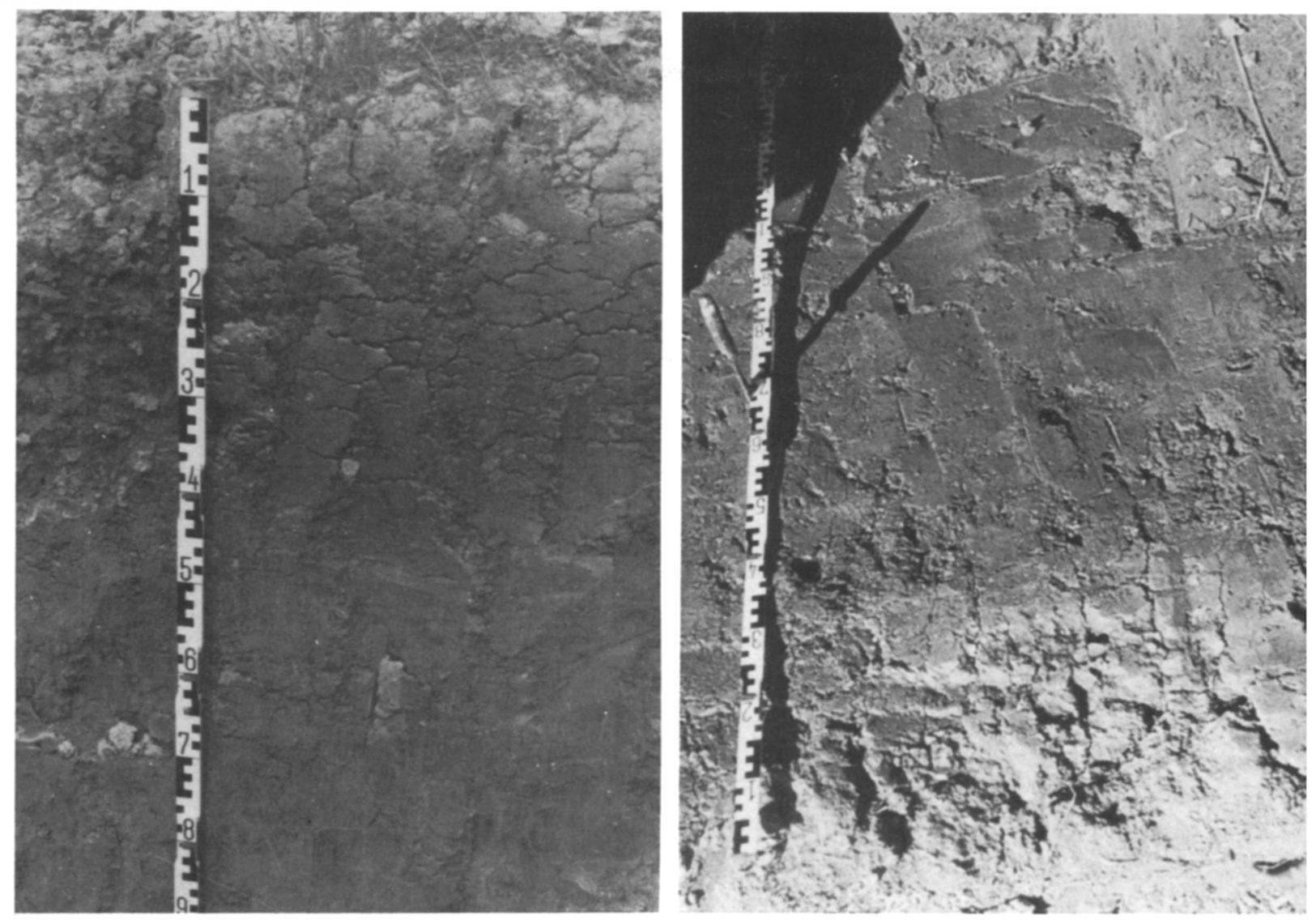

Profile 2

Profile 3

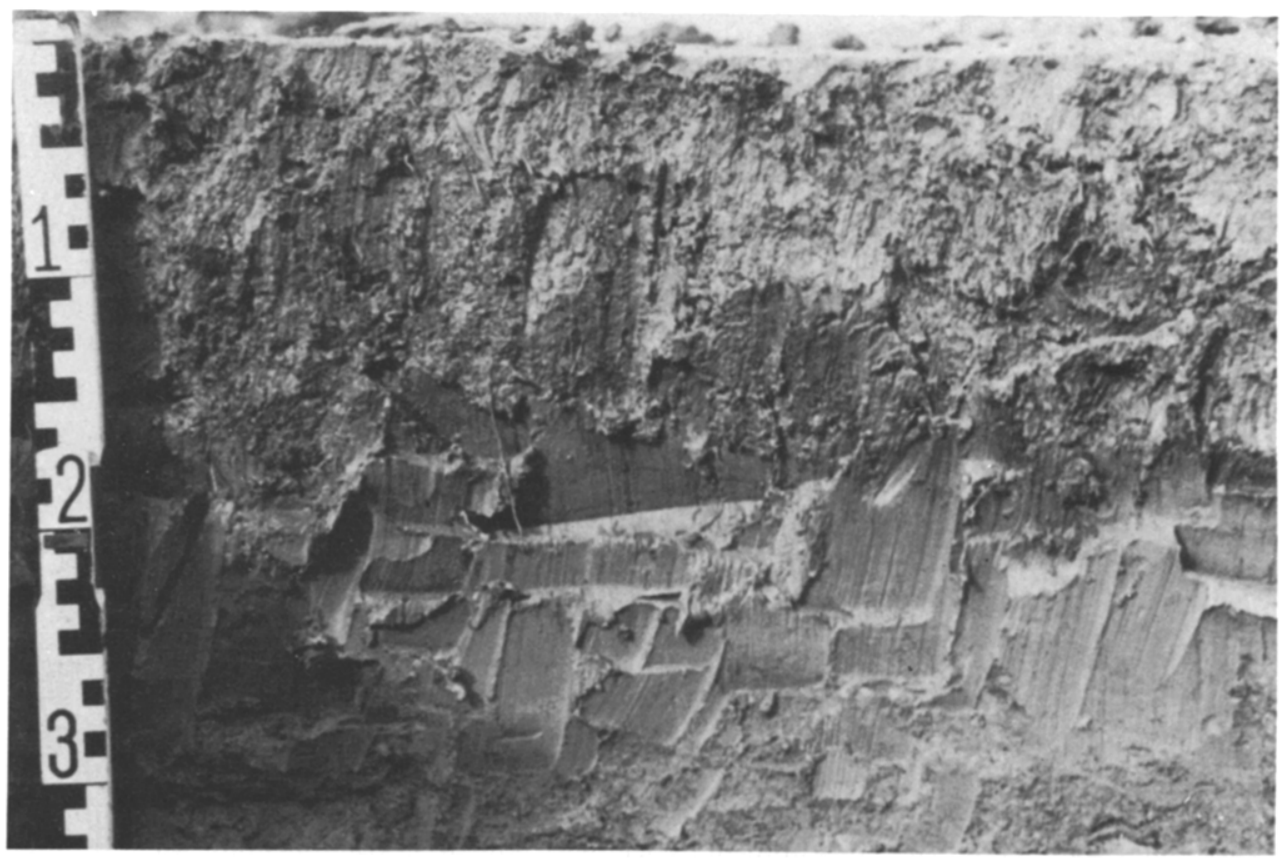




\subsection{METHODS}

The following analyses have been done:

In undisturbed samples:

- Hydraulic conductivity in saturated status in constant water head permeameter, following the technique of FLANNERY \& KIRKHAM (1964) in an apparatus designed by MARTIN ARANDA (1973).

- Bulk density $\left(D_{b}\right)$ (from weight/volume ratio of core samples).

- Coefficient of linear extensibility (COLE) (from volume difference in sample cores, from field capacity to air dry).

In undisturbed subsamples:

- $\mathrm{pF}$ values $(0,0.5,1.0,1.5,2.0,2.5,3.7$ and 4.2$)$. The range $0-1.5$ by suction in fritted glass plates following a technique similar to that of VOMOCIL (1965) and 2.0-4.2 by pressure in ceramic plates, in Richards chambers (RICHARDS 1948).

In disturbed samples:

- Granulometric analyses, by chain hydrometer (DE LEENHEER et al. 1965).

- $\mathrm{CaCO}_{3}$ content, in Bernard's calcimeter (HIDALGO \& CANDELA 1958).

- Salinity (following the U.S. Salinity Lab. Methods, RICHARDS 1954).

- Organic matter content, following Walkley and Black method, modified (PIPER 1950).

- Particle density $\left(\mathrm{D}_{\mathrm{p}}\right)$ (by the use of a helium-air pycnometer, Micromeritics, mod. 1302).

\section{RESULTS AND DISCUSSION}

\subsection{PROFILE DESCRIPTIONS}

\section{PROFILE 1}

Classification: Fluvaquent

Location: Lebrija (Sevilla). Farm "La Señuela". Section III of Marismas.

Longitude: $6^{\circ} 10^{\prime} 25^{\prime \prime} \mathrm{W}$.; Latitude: $36^{\circ} 56^{\prime} 30^{\prime \prime} \mathrm{N}$.

Altitude: $2 \mathrm{~m}$; Topography: Alluvial plain; Slope: $1 \%$.

Parent material: Colluvial clayey deposits.

Vegetation and use: Natural pasture (hallophytes).

$\mathrm{A}_{1} \quad 0-10 \mathrm{~cm} \quad$ Dark gray brown (10 YR 4/2) moist, light gray (10 YR 7/2) dry; clayey; medium to large moderate to well developed, subangular blocky structure, tending to prismatic; very plastic and adhesive wet, firm moist, very hard dry; calcareous; salt efflorescence on dry aggregates surfaces; sharp smooth boundary.

$\mathrm{B}_{11} \mathrm{~g} \quad 10-37 \mathrm{~cm} \quad$ Dark gray brown (10 YR 4/2) moist; clayey; slightly developed, massive structure with tendency to blocky-prismatic; very plastic and adhesive wet, firm moist; diffuse red-brown mottling increasing with depth; abundant radicular saline micelium partially hardened; calcareous; gradual smooth boundary.

$\mathrm{B}_{12} \mathrm{~g} \quad 37-56 \mathrm{~cm} \quad$ Diffuse dominant variegate colour, dark gray $(5 Y 4 / 1)$ with dark brown mottles (7.5 YR 4/4); clayey; massive structure with a tendency to weakly developed blocky-prismatic; very plastic and adhesive wet, firm moist; calcareous; abundant anastomosed tubular, salt micelium; gradual smooth boundary.

$\mathrm{Cg} \quad 56-110 \mathrm{~cm} \quad$ Dark brown variegate colour (7.5 YR 4/4) moist and dark gray (5 Y 4/1) moist; clayey; large blocky structure; plastic and adhesive wet, firm moist; calcareous; abundant white salt micelium with gray mottles.

PROFILE 2

Classification: Haplaquept.

Location: Lebrija (Sevilla). Subsection B, Section III of Marismas. 
Longitude: $6^{\circ} 4^{\prime} 40^{\prime \prime} \mathrm{W}$; Latitude: $36^{\circ} 57^{\prime} 50^{\prime \prime} \mathrm{N}$.

Altitude: $5 \mathrm{~m}$; Topography: Alluvial plain; Slope: $2 \%$.

Parent material: Colluvial clayey deposits.

Vegetation and use: Wheat (after drainage).

Ap $\quad 0-20 \mathrm{~cm}$

Gray to gray brown (10 YR 5.5/2) dry, dark gray brown (10 YR 4/2) moist; clayey; large columnar prismatic blocky structure, strongly developed in the top $10 \mathrm{~cm}$, with large subangular blocky substructure, subconcoideous corrugated surface fracture; plastic and adhesive wet, firm and friable moist, very hard dry; strongly calcareous; frequent small gypsum concretions; slightly wavy boundary.

$\mathrm{Bg} \quad 20-40 \mathrm{~cm} \quad$ Diffuse variegated colour, finely mottled, brown (10 YR 5/3) matrix moist; clayey; massive structure breaking up into prismatic columnar blocks, a little smaller than in previous horizon; plastic and adhesive wet, firm to friable moist, hard dry; strongly calcareous; gypsum micelium and concretions concentrated in a layer between 25 and $35 \mathrm{~cm}$ depth, diffuse boundary.

$\mathrm{C}_{1} \mathrm{~g} \quad 40-80 \mathrm{~cm} \quad$ More defined variegated colour, on brown (10 YR 5/3) matrix, moist; with gray-dark gray (5 Y 3.5/1) and dark gray brown (10 YR 4/2) streaks moist; clayey; massive structure which breaks up into prismatic-columnar or subangular-prismatic blocks; plastic and adhesive wet, very firm moist; frequent gypsum micelium, concentrated at the horizon base, forming a more diffuse layer than before; more frequent, larger and more defined ferruginous mottles and concretions with depth; gray streaks along channels and galleries; strongly calcareous; gradual boundary.

$\mathrm{C}_{2} \mathrm{~g} \quad 80-220 \mathrm{~cm} \quad$ variegated colour, with more defined and larger mottles than in previous horizon, gray (5 Y 6/1), brown-dark brown (7.5 YR 4/4) and brown (7.5 YR 5/4); clayey; massive structure with tendency to medium blocky; very plastic and adhesive wet, very firm moist; frequent ferruginous concretions; calcareous; gradual boundary.

IIC $_{1} \quad 220-350 \mathrm{~cm} \quad$ Dark gray (10 YR 4/1) moist; clayey to clay loam; massive structure; very plastic and adhesive wet, very firm moist; compact; calcareous; diffuse boundary.

IIC $\quad 350-\quad \mathrm{cm} \quad$ Same characteristics as before, but with the presence of abundant fragments of fine shells.

PROFILE 3

Classification: Haplaquept.

Location: Las Cabezas de San Juan (Sevilla). Farm "Quevedo - COTEMSA".

Longitude: $6^{\circ} 0^{\prime} 0^{\prime \prime} \mathrm{W}$; L Latitude: $37^{\circ} 4^{\prime} 10^{\prime \prime} \mathrm{N}$.

Altitude: $10 \mathrm{~m}$; Topography: Alluvial plain; Slope: $1 \%$.

Parent material: Colluvial clayey deposits.

Vegetation and use: Rice (20 years).

$\mathrm{Ap}_{1} \quad 0-5 \mathrm{~cm}$

Dark gray brown (2.5 Y 4.5/2) moist, with fine diffuse yellowish brown streaks (10 YR 5/4), coincident with fine radicular ferruginous channels, small black mottles or concretions; clayey; weakly developed, medium crumby structure; plastic and adhesive moist; calcareous; abrupt broken boundary.

$\mathrm{Ap}_{2} \quad 5-20 \mathrm{~cm} \quad$ dark to very dark gray (7.5 YR 3.5/0) moist; clayey; massive, without visible aggregates; very plastic and adhesive; calcareous; abrupt somewhat wavy boundary

Bg 20-30 cm Brown to dark brown (10 YR 4/3) moist, with fine isolated gray streaks (5 Y $5 / 1)$ and abundant very diffuse yellowish brown mottles (10 YR 5/4) and some very small black mottles or concretions; clayey; massive; very plastic and adhesive; very compact; very firm moist; calcareous; gradual boundary.

$\mathrm{C}_{1} \mathrm{~g} \quad 30-55 \mathrm{~cm} \quad$ Diffuse variegate colour, on brown to dark brown matrix (10 YR 4/3) moist, with fine and medium long gray streaks $(2.5 \mathrm{Y} 5 / 0)$ and diffuse brown to dark brown mottles (7.5 YR 4/4), parallel to gray streaks; clayey; weakly developed subangular blocky structure tending to massive; very plastic and adhesive wet; very firm moist; frequent small concretions; compact; calcareous; gradual boundary. 
$\mathrm{C}_{2} \mathrm{~g} \quad 55-100 \mathrm{~cm} \quad$ Very diffuse variegate colour on brown matrix (7.5 YR 5/4) moist, with ferruginous very dark medium-size mottles or concretions, and frequent hard and fragile crystaline gypsum micelium or tubulum, filing 2-3 mm diameter root voids; clayey; massive; very plastic and adhesive; calcareous; diffuse boundary.

$\mathrm{C}_{3} \mathrm{~g} \quad 100-\mathrm{cm} \quad$ Variegate brown (10 YR 5/3) and strong brown (7.5 YR 5/6) colour moist, with fine gray streaks, following the small radicular channels, bordered by strong brown mottles (7.5 YR 5/6) moist. Same general characteristics as before. Micelium forms a polygonal interlocking network; frequent ferruginous dark nodules.

\begin{abstract}
PRO FIL E 4
Classification: Chromoxerert.

Location: Lebrija (Sevilla), Lake "Val de Ojo".

Longitude: $6^{\circ} 3^{\prime} 30^{\prime \prime} \mathrm{W}$; Latitude: $36^{\circ} 57^{\prime} 5^{\prime \prime} \mathrm{N}$.

Altitude: $8 \mathrm{~m}$; Topography: Alluvial plain; Slope: $2 \%$.

Parent material: Marly-saline alluvial deposits.

Vegetation and use: Natural pasture (hallophytes).

$\mathrm{A}_{11} \quad 0-10 \mathrm{~cm} \quad$ Dark brown (7.5 YR 5/2) moist, brown (7.5 YR 5/2) dry, clayey; medium to large granular structure, strongly developed in the surface and moderately in the base of horizon; plastic and adhesive wet, friable moist, very hard dry; calcareous; sharp, smooth boundary.

A 12 10-20 cm Brown to dark brown (7.5 YR 4/2) moist; clayey; medium to fine moderately developed, subangular blocky structure; plastic and adhesive wet, friable to firm moist, hard dry; calcareous; gradual, smooth boundary. Cracks each $20 \mathrm{~cm}$ are appreciated.

B 20-34 cm Brown to dark brown (7.5 YR 4/3) moist, dark brown (7.5 YR 5/3) dry; clayey large blocky-prismatic peds structure, with corrugated faces and salt efflorescence; plastic and adhesive wet, very firm moist, very hard dry; aggregates show tendency to concoideous fracture; calcareous, with some small lime nodules, and also very isolated medium ones; gradual boundary. Cracks are observed.

$\mathrm{BC} \quad 34-50 \mathrm{~cm} \quad$ Same colour and texture as above, strongly developed large blocky structure tending to prismatic; somewhat concoideous fracture and shiny faces; plastic and adhesive wet, very firm moist, very hard dry; calcareous, with abundant calcosaline concretions; gradual boundary. Appreciable cracks.

$\mathrm{C}_{1} \quad 50-80 \mathrm{~cm} \quad$ Brown to dark brown (7.5 YR 4/3) with diffuse gray mottles (7.5 YR 5/1) and small strong brown still more diffuse mottles (7.5 YR 4/4) moist; clayey; massive; very plastic and adhesive wet, very firm moist; compact; frequent calcosaline concretions or micelium; gradual boundary.

$\mathrm{C}_{2} \quad 80-105 \mathrm{~cm} \quad$ Same general characteristics as above; tendency to blocky structure with shiny face aggregates; more plastic; calcosaline concretions; diffuse boundary.

$\mathrm{C}_{3} \quad 105-145 \mathrm{~cm} \quad$ Dark brown (7.5 YR 4/2) moist, with frequent very small white calcosaline concretions, uniformly distributed in the soil; clayey; massive structure tending to blocky with shiny faces; plastic and adhesive wet, firm moist; compact; calcareous; sharp boundary.

$\mathrm{C}_{4} \quad 145-200 \mathrm{~cm} \quad$ Brown (7.5 YR 4.5/2) moist; less frequent calcosaline concretions; clayey; massive; very plastic and adhesive wet, firm to very firm moist; compact; calcareous.
\end{abstract}

PROFILE 5

Classification: Pelloxerert-Haplaquept.

Location: Sevilla. Farm "Micones".

Longitude: $6^{\circ} 5^{\prime \prime} 2^{\prime \prime}$ W. Latitude: $36^{\circ} 52^{\prime} 2^{\prime \prime} \mathrm{N}$.

Altitude: $2 \mathrm{~m}$; Topography: Alluvial plain; Slope: $2 \%$.

Parent material: Colluvial clayey deposits.

Vegetation and use: Wheat and barley ( 5 years). 
Gray (10 YR 6/1) dry; gray (10 YR 5/1) moist; clayey; medium to fine well developed subangular blocky structure; large cracks $20 \mathrm{~cm}$ long, $2-3 \mathrm{~cm}$ width, each $10-15 \mathrm{~cm}$, configurating large prismatic-columnar blocks; very plastic and adhesive wet, very firm moist, extremely hard dry; lime reaction; sharp smooth boundary.

$\mathrm{Ap}_{2} \quad 7.21 \mathrm{~cm} \quad$ Dark gray (10 YR 4/1) dry, gray (10 YR 5/1) moist; clayey; fine to medium moderate to well developed subangular blocky structure; plastic and adhesive wet, firm moist, very hard dry; no concretions; diffuse mottles; abrupt and smooth boundary.

B $\quad 21-50 \mathrm{~cm} \quad$ Gray (10 YR 5/1) moist; clayey; massive; moderately vertic with shiny concoideous fractures; plastic and adhesive wet, firm moist; slikensides; no concretions; gradual boundary.

BC $\quad 50-70 \mathrm{~cm} \quad$ Gray brown matrix (10 YR 5/2) moist, with diffuse mottles, brown (10 YR 4/3) to gray brown (2.5 Y 5/2) streaks; clayey; plastic and adhesive wet; friable to firm moist; compact; shiny surfaces; concoideous fractures; gradual to diffuse boundary.

$\mathrm{Cg}_{1} \quad 70-130 \mathrm{~cm} \quad$ Dark brown matrix (10 YR 4/3), dominant brown (7.5 YR 4/2) or brown (10 YR 5/3) streaks; clayey; massive; weakly developed large blocky structure shiny concoideous fracture; abundant saline tubular streaks increasing with depth; slight discontinuous diffuse dark $2 \mathrm{~mm}$ mottling; plastic and adhesive wet; compact; lime reaction; diffuse boundary.

$\mathrm{Cg}_{2}$ 130- $\mathrm{cm} \quad$ Brown matrix (10 YR 5/3) moist; with isolated yellowish brown (10 YR 5/8) mottles; other characteristics as above.

\section{PROFILE 6}

Classification: Pelloxerert.

Location: Utrera (Sevilla). Farm "Torre Marismas".

Longitude: $5^{\circ} 53^{\prime} 40^{\prime \prime} \mathrm{W}$; : Latitude: $37^{\circ} 2^{\prime} 35^{\prime \prime} \mathrm{N}$.

Altitude: $10 \mathrm{~m}$; Topography: Plain; Slope: $2 \%$.

Parent material: Calcareous marl deposits.

Vegetation and use: Wheat and sunflower.

$\mathrm{Ap}_{1} \quad 0-20 \mathrm{~cm}$

Dark gray (10 YR 4/1), very dark gray (10 YR 3/1) moist; clayey; medium subangular blocky structure, strongly developed on the surface, when dry, with tendency to crumby moist; concoideous fracture aggregates; plastic and adhesive wet, firm moist, hard dry; abundant very small calcareous concretions gradual boundary.
$\mathrm{Ap}_{2} \quad 20-40 \mathrm{~cm}$
Dark gray (10 YR 4/1) moist; clayey; weakly developed medium to fine crumby structure, tending to massive; shiny concoideous fracture aggregates; plastic and adhesive wet, firm moist, hard dry; frequent very small calcareous concretions; gradual boundary.
$B_{11} \quad 40-60 \mathrm{~cm}$
Dark gray (10 YR 4/1) moist; clayey; massive structure, with a tendency to form small and medium shiny-surface blocky aggregates; plastic and adhesive wet, firm moist, very hard dry; scarce very small calcareous concretions; gradual boundary.
$B_{12} \quad 60-80 \mathrm{~cm} \quad$ Dark gray (10 YR 4/1) moist; clayey; weakly developed blocky structure; massive in fresh cuts; plastic and adhesive wet, firm moist, very hard dry; very scarce, very small, calcareous concretions; gradual smooth boundary.
$\mathrm{B}_{2} \quad 80-120 \mathrm{~cm}$ Dark to very dark gray (10 YR 3/1) moist; clayey; structure formed by large blocky peds with slikensides; massive in fresh cuts; plastic and adhesive wet, very firm moist, very hard dry; very small isolated calcareous concretions; gradual boundary.
BC 120- cm Dark gray brown (10 YR 4/2), with diffuse dark greyish streaks (10 YR 4/1) and brown (10 YR 5/3) moist; clayey; massive; without visible aggregates in fresh cuts; plastic and adhesive wet, firm moist; compact; lime reaction stron- ger than in previous horizons. 
Tab. 1: GENERAL PHYSICAL PROPERTIES

\begin{tabular}{|c|c|c|c|c|c|c|c|c|c|}
\hline \multirow{2}{*}{ Profile } & \multirow{2}{*}{$\begin{array}{l}\text { Depth } \\
\mathrm{cm}\end{array}$} & \multicolumn{4}{|c|}{ Particle size (mm, \%) } & \multirow{2}{*}{$\begin{array}{l}\mathrm{Dp} \\
\mathrm{g} / \mathrm{cm}^{3}\end{array}$} & \multirow{2}{*}{$\begin{array}{l}\mathrm{Db} \\
\mathrm{g} / \mathrm{cm}^{3}\end{array}$} & \multirow{2}{*}{$\begin{array}{l}\mathrm{K} \\
\mathrm{cm} / \mathrm{h}\end{array}$} & \multirow{2}{*}{$\begin{array}{l}\text { COLE } \\
\times 10^{2} \\
\end{array}$} \\
\hline & & $>0.2$ & $0.2-0.02$ & $0.02-0.002$ & $<0.002$ & & & & \\
\hline 1 & $\begin{array}{c}0-10 \\
10-20 \\
20-30 \\
30-50 \\
50-70 \\
70-90 \\
90-110\end{array}$ & $\begin{array}{l}0.5 \\
0.5 \\
0.5 \\
0.5 \\
1.0 \\
0.5 \\
1.0\end{array}$ & $\begin{array}{l}1.0 \\
1.5 \\
1.5 \\
1.5 \\
2.5 \\
2.5 \\
3.5\end{array}$ & $\begin{array}{l}27.5 \\
35.0 \\
29.0 \\
25.0 \\
33.5 \\
62.5 \\
42.0\end{array}$ & $\begin{array}{l}71.0 \\
62.5 \\
69.0 \\
73.0 \\
63.0 \\
34.5 \\
53.0\end{array}$ & $\begin{array}{l}2.70 \\
2.70 \\
2.73 \\
2.74 \\
2.74 \\
2.77 \\
2.77\end{array}$ & $\begin{array}{l}1.43 \\
1.44 \\
1.44 \\
1.39 \\
1.43 \\
1.45 \\
1.30\end{array}$ & $\begin{array}{l}0.5 \\
0.2 \\
0.1 \\
0.1 \\
0.1 \\
0.5 \\
0.1\end{array}$ & $\begin{array}{r}7.0 \\
7.8 \\
7.1 \\
9.4 \\
6.7 \\
7.9 \\
11.3\end{array}$ \\
\hline 2 & $\begin{array}{c}0-10 \\
10-20 \\
20-30 \\
30-50 \\
50-70 \\
70-90 \\
90-110\end{array}$ & $\begin{array}{l}0.5 \\
0.5 \\
0.5 \\
0.5 \\
0.5 \\
0.5 \\
0.5\end{array}$ & $\begin{array}{l}1.5 \\
2.5 \\
2.5 \\
0.5 \\
0.5 \\
0.5 \\
1.0\end{array}$ & $\begin{array}{l}28.0 \\
29.0 \\
29.0 \\
36.0 \\
29.0 \\
34.0 \\
33.5\end{array}$ & $\begin{array}{l}70.0 \\
68.0 \\
68.0 \\
63.0 \\
70.0 \\
65.0 \\
65.0\end{array}$ & $\begin{array}{l}2.72 \\
2.74 \\
2.75 \\
2.75 \\
2.78 \\
2.79 \\
2.79\end{array}$ & $\begin{array}{l}1.30 \\
1.37 \\
1.26 \\
1.16 \\
1.26 \\
1.31 \\
1.27\end{array}$ & $\begin{array}{r}0.2 \\
<0.1 \\
<0.1 \\
\quad 1.5 \\
<0.1 \\
<0.1 \\
<0.1\end{array}$ & $\begin{array}{r}12.8 \\
11.1 \\
11.6 \\
8.0 \\
11.4 \\
10.6 \\
10.0\end{array}$ \\
\hline 3 & $\begin{array}{c}0-10 \\
10-20 \\
20-30 \\
30-50 \\
50-70 \\
70-90 \\
90-110\end{array}$ & $\begin{array}{l}0.5 \\
0.5 \\
0.5 \\
0.5 \\
0.5 \\
0.5 \\
1.0\end{array}$ & $\begin{array}{l}1.5 \\
1.5 \\
1.5 \\
2.0 \\
2.0 \\
2.0 \\
2.0\end{array}$ & $\begin{array}{l}28.0 \\
28.0 \\
31.0 \\
27.0 \\
30.5 \\
32.0 \\
30.0\end{array}$ & $\begin{array}{l}69.5 \\
70.0 \\
67.0 \\
70.5 \\
67.0 \\
65.5 \\
67.0\end{array}$ & $\begin{array}{l}2.72 \\
2.70 \\
2.75 \\
2.80 \\
2.79 \\
2.80 \\
2.80\end{array}$ & $\begin{array}{l}0.96 \\
1.01 \\
1.27 \\
1.31 \\
1.20 \\
1.10 \\
1.10\end{array}$ & $\begin{array}{r}0.3 \\
<0.1 \\
0.3 \\
<0.1 \\
<0.1 \\
<0.1 \\
<0.1\end{array}$ & $\begin{array}{r}17.1 \\
18.8 \\
13.0 \\
8.8 \\
11.5 \\
14.0 \\
13.8\end{array}$ \\
\hline 4 & $\begin{array}{c}0-10 \\
10-20 \\
20-30 \\
30-50 \\
50-70 \\
70-90 \\
90-110\end{array}$ & $\begin{array}{l}1.0 \\
0.5 \\
0.5 \\
0.5 \\
0.5 \\
0.5 \\
0.5\end{array}$ & $\begin{array}{l}3.0 \\
2.5 \\
2.5 \\
2.0 \\
1.5 \\
1.5 \\
2.5\end{array}$ & $\begin{array}{l}39.0 \\
32.0 \\
28.0 \\
39.0 \\
33.0 \\
31.5 \\
34.5\end{array}$ & $\begin{array}{l}56.5 \\
65.0 \\
69.0 \\
58.5 \\
65.0 \\
67.5 \\
62.5\end{array}$ & $\begin{array}{l}2.70 \\
2.71 \\
2.74 \\
2.76 \\
2.77 \\
2.79 \\
2.78\end{array}$ & $\begin{array}{l}1.39 \\
1.32 \\
1.40 \\
1.33 \\
1.35 \\
1.37 \\
1.32\end{array}$ & $\begin{array}{r}0.1 \\
0.1 \\
<0.1 \\
<0.1 \\
0.1 \\
0.1 \\
<0.1\end{array}$ & $\begin{array}{r}9.7 \\
8.6 \\
7.8 \\
10.0 \\
7.7 \\
7.8 \\
9.7\end{array}$ \\
\hline 5 & $\begin{array}{c}0-10 \\
10-25 \\
25-35 \\
35-50 \\
50-80 \\
90-110\end{array}$ & $\begin{array}{l}0.5 \\
0.5 \\
0.5 \\
0.5 \\
0.5 \\
0.5\end{array}$ & $\begin{array}{l}2.5 \\
2.5 \\
0.5 \\
1.0 \\
1.5 \\
1.0\end{array}$ & $\begin{array}{l}39.5 \\
41.0 \\
41.0 \\
39.0 \\
41.0 \\
39.5\end{array}$ & $\begin{array}{l}57.0 \\
56.0 \\
58.0 \\
59.5 \\
57.0 \\
58.5\end{array}$ & $\begin{array}{l}2.66 \\
2.70 \\
2.70 \\
2.71 \\
2.72 \\
2.74\end{array}$ & $\begin{array}{l}1.16 \\
1.13 \\
1.23 \\
1.27 \\
1.23 \\
1.26\end{array}$ & $\begin{array}{l}3.8 \\
2.7 \\
0.3 \\
0.3 \\
0.2 \\
0.3\end{array}$ & $\begin{array}{r}14.2 \\
14.7 \\
14.7 \\
12.4 \\
9.7 \\
13.5\end{array}$ \\
\hline 6 & $\begin{array}{c}0-10 \\
10-20 \\
20-30 \\
30-50 \\
50-70 \\
70-90 \\
90-110\end{array}$ & $\begin{array}{l}2.0 \\
2.5 \\
2.0 \\
2.0 \\
3.5 \\
3.0 \\
3.0\end{array}$ & $\begin{array}{l}15.5 \\
15.0 \\
16.0 \\
16.5 \\
16.5 \\
16.0 \\
12.0\end{array}$ & $\begin{array}{l}23.0 \\
23.0 \\
22.5 \\
22.5 \\
21.0 \\
22.5 \\
20.0\end{array}$ & $\begin{array}{l}59.0 \\
59.0 \\
59.0 \\
58.5 \\
59.0 \\
58.5 \\
65.0\end{array}$ & $\begin{array}{l}2.75 \\
2.72 \\
2.74 \\
2.78 \\
2.79 \\
2.73 \\
2.76\end{array}$ & $\begin{array}{l}1.15 \\
1.33 \\
1.25 \\
1.23 \\
1.29 \\
1.30 \\
1.31\end{array}$ & $\begin{array}{l}6.5 \\
0.1 \\
0.1 \\
0.1 \\
0.1 \\
1.5 \\
0.1\end{array}$ & $\begin{array}{l}14.3 \\
12.6 \\
13.0 \\
13.0 \\
12.8 \\
12.4 \\
12.4\end{array}$ \\
\hline
\end{tabular}

\subsection{GENERAL PHYSICAL PROPERTIES}

Particle-size range percentages, particle density $\left(D_{p}\right)$, bulk density $\left(D_{b}\right)$, hydraulic conductivity $(\mathrm{K})$ and coefficient of linear extensibility (COLE) for the different profiles are given in table 1 . 
Tab. 2: WATER RETENTION (weight \%)

\begin{tabular}{|c|c|c|c|c|c|c|c|c|c|}
\hline Protile & $\begin{array}{l}\text { Depth } \\
\mathrm{cm}\end{array}$ & 0 & 0.5 & 1.0 & 1.5 & $\begin{array}{l}\mathrm{pF} \\
2.0 \\
\end{array}$ & 2.5 & 3.7 & 4.2 \\
\hline 1 & $\begin{array}{l}0-10 \\
10-20 \\
20-30 \\
30-50 \\
50-70 \\
70-90 \\
90-110\end{array}$ & $\begin{array}{l}37.2 \\
36.2 \\
40.1 \\
43.4 \\
40.1 \\
39.4 \\
44.1\end{array}$ & $\begin{array}{l}35.6 \\
34.9 \\
38.8 \\
40.6 \\
38.3 \\
38.3 \\
42.7\end{array}$ & $\begin{array}{l}34.2 \\
33.8 \\
38.0 \\
39.3 \\
37.2 \\
37.7 \\
41.7\end{array}$ & $\begin{array}{l}33.5 \\
33.7 \\
37.5 \\
39.0 \\
37.0 \\
37.1 \\
41.3\end{array}$ & $\begin{array}{l}32.8 \\
33.6 \\
37.0 \\
38.7 \\
36.8 \\
36.8 \\
40.9\end{array}$ & $\begin{array}{l}30.6 \\
31.9 \\
34.8 \\
36.3 \\
34.2 \\
35.3 \\
39.7\end{array}$ & $\begin{array}{l}27.1 \\
29.7 \\
31.5 \\
32.4 \\
30.1 \\
31.7 \\
37.3\end{array}$ & $\begin{array}{l}26.7 \\
28.7 \\
30.7 \\
30.8 \\
28.2 \\
30.6 \\
36.5\end{array}$ \\
\hline 2 & $\begin{array}{c}0-10 \\
10-20 \\
20-30 \\
30-50 \\
50-70 \\
70-90 \\
90-110\end{array}$ & $\begin{array}{l}44.8 \\
44.5 \\
43.8 \\
46.8 \\
50.6 \\
47.8 \\
47.2\end{array}$ & $\begin{array}{l}43.8 \\
43.7 \\
43.3 \\
46.6 \\
50.1 \\
47.5 \\
47.0\end{array}$ & $\begin{array}{l}42.8 \\
42.6 \\
42.3 \\
44.6 \\
49.4 \\
46.5 \\
46.0\end{array}$ & $\begin{array}{l}40.8 \\
40.5 \\
41.2 \\
43.8 \\
48.5 \\
45.7 \\
44.9\end{array}$ & $\begin{array}{l}39.6 \\
39.5 \\
40.2 \\
42.7 \\
46.3 \\
43.5 \\
42.8\end{array}$ & $\begin{array}{l}38.0 \\
38.1 \\
38.7 \\
40.8 \\
43.4 \\
41.6 \\
40.7\end{array}$ & $\begin{array}{l}34.9 \\
34.7 \\
35.8 \\
37.0 \\
40.0 \\
38.8 \\
37.4\end{array}$ & $\begin{array}{l}33.2 \\
33.0 \\
33.8 \\
35.9 \\
38.4 \\
36.1 \\
35.8\end{array}$ \\
\hline 3 & $\begin{array}{c}0-10 \\
10-20 \\
20-30 \\
30-50 \\
50-70 \\
70-90 \\
90-110\end{array}$ & $\begin{array}{l}63.8 \\
62.1 \\
42.0 \\
43.5 \\
51.6 \\
60.4 \\
54.1\end{array}$ & $\begin{array}{l}63.2 \\
60.1 \\
41.3 \\
42.3 \\
50.6 \\
60.0 \\
53.7\end{array}$ & $\begin{array}{l}63.1 \\
59.8 \\
41.0 \\
41.9 \\
50.2 \\
59.7 \\
53.4\end{array}$ & $\begin{array}{l}62.5 \\
59.6 \\
40.6 \\
41.1 \\
49.9 \\
58.7 \\
52.9\end{array}$ & $\begin{array}{l}60.0 \\
57.8 \\
40.5 \\
39.3 \\
47.0 \\
55.1 \\
51.2\end{array}$ & $\begin{array}{l}55.0 \\
52.3 \\
39.9 \\
38.2 \\
45.2 \\
53.6 \\
50.4\end{array}$ & $\begin{array}{l}47.5 \\
46.9 \\
35.9 \\
33.3 \\
38.6 \\
46.7 \\
44.0\end{array}$ & $\begin{array}{l}44.0 \\
44.9 \\
34.4 \\
32.2 \\
36.8 \\
45.4 \\
42.2\end{array}$ \\
\hline 4 & $\begin{array}{c}0-10 \\
10-20 \\
20-30 \\
30-50 \\
50-70 \\
70-90 \\
90-110\end{array}$ & $\begin{array}{l}38.3 \\
32.6 \\
34.2 \\
38.0 \\
41.3 \\
44.1 \\
47.1\end{array}$ & $\begin{array}{l}36.8 \\
31.6 \\
33.5 \\
37.5 \\
40.9 \\
43.6 \\
46.7\end{array}$ & $\begin{array}{l}36.6 \\
31.1 \\
32.7 \\
37.0 \\
40.6 \\
42.8 \\
46.3\end{array}$ & $\begin{array}{l}36.2 \\
30.6 \\
32.4 \\
36.8 \\
39.9 \\
42.5 \\
46.0\end{array}$ & $\begin{array}{l}36.0 \\
30.2 \\
31.5 \\
36.0 \\
39.3 \\
41.1 \\
45.4\end{array}$ & $\begin{array}{l}34.9 \\
29.8 \\
29.9 \\
33.9 \\
36.3 \\
38.8 \\
43.7\end{array}$ & $\begin{array}{l}30.3 \\
26.6 \\
26.6 \\
30.0 \\
31.6 \\
34.6 \\
39.0\end{array}$ & $\begin{array}{l}29.4 \\
26.0 \\
26.0 \\
28.6 \\
29.4 \\
32.9 \\
37.4\end{array}$ \\
\hline 5 & $\begin{array}{l}0-10 \\
10-25 \\
25-35 \\
35-50 \\
50-80 \\
90-110\end{array}$ & $\begin{array}{l}48.6 \\
47.7 \\
45.9 \\
46.1 \\
47.6 \\
48.0\end{array}$ & $\begin{array}{l}47.9 \\
47.0 \\
45.3 \\
45.5 \\
47.1 \\
47.3\end{array}$ & $\begin{array}{l}47.3 \\
46.1 \\
45.1 \\
45.2 \\
46.8 \\
47.0\end{array}$ & $\begin{array}{l}46.6 \\
45.3 \\
44.7 \\
44.3 \\
45.9 \\
46.8\end{array}$ & $\begin{array}{l}45.1 \\
44.0 \\
42.9 \\
42.9 \\
42.9 \\
45.0\end{array}$ & $\begin{array}{l}44.0 \\
40.7 \\
39.4 \\
41.0 \\
41.0 \\
42.9\end{array}$ & $\begin{array}{l}35.7 \\
34.6 \\
33.4 \\
36.2 \\
34.5 \\
36.6\end{array}$ & $\begin{array}{l}35.0 \\
34.0 \\
32.7 \\
34.8 \\
33.4 \\
35.6\end{array}$ \\
\hline 6 & $\begin{array}{c}0-10 \\
10-20 \\
20-30 \\
30-50 \\
50-70 \\
70-90 \\
90-110\end{array}$ & $\begin{array}{l}52.6 \\
49.1 \\
52.6 \\
46.6 \\
45.9 \\
46.1 \\
44.4 \\
\end{array}$ & $\begin{array}{l}51.2 \\
48.1 \\
51.1 \\
44.8 \\
44.8 \\
44.5 \\
43.2 \\
\end{array}$ & $\begin{array}{l}49.8 \\
47.1 \\
49.6 \\
43.3 \\
43.3 \\
41.7 \\
41.8 \\
\end{array}$ & $\begin{array}{l}46.4 \\
43.3 \\
44.6 \\
39.1 \\
38.6 \\
37.8 \\
38.3 \\
\end{array}$ & $\begin{array}{l}42.4 \\
40.4 \\
42.7 \\
36.3 \\
35.8 \\
34.9 \\
35.4\end{array}$ & $\begin{array}{l}40.1 \\
38.2 \\
38.8 \\
32.6 \\
32.6 \\
32.1 \\
32.7 \\
\end{array}$ & $\begin{array}{l}35.7 \\
34.5 \\
34.4 \\
28.4 \\
29.3 \\
29.7 \\
29.9 \\
\end{array}$ & $\begin{array}{l}34.9 \\
33.8 \\
33.2 \\
27.2 \\
28.2 \\
28.4 \\
28.8\end{array}$ \\
\hline
\end{tabular}

Particle-size relative percentages are always typical of heavy clay soils, although certain variations are observed between profiles and also between different horizons in a given profile. In profile 1 , in relation to the others, a noticeable reduction in clay content is observed, in favour of silt, between 70 and $90 \mathrm{~cm}$ depth, while in profiles 2,3 and 5 there is little change. Variations within a given profile, in profiles 1 and 4 , could be explained considering they are 
the most natural and the nearest to the river (Guadalquivir and Salado, respectively) being therefore recent sediments. Profiles 3 and 6 (to a depth of $90 \mathrm{~cm}$ ) are the most homogeneous; in both examples this is a consequence of the continuous agronomical use of these soils (MORENO et al. 1979): in the first case with a rice crop, in the second with winter and summer crops, usual for vertisols in the area (wheat, barley; cotton, sunflower, com).

Bulk density $\left(D_{b}\right)$ is another parameter linked to the agronomical status of soils. The highest values correspond to profiles 1 and 4 (soils maintained in natural condition), while the lowest values correspond to top layers of profiles 3,5 and 6 . Profile 2 represents an intermediate situation because of its recent use after drainage.

Hydraulic conductivity (K) (see table 1), as usual in this type of soil, is low. Only the upper $10 \mathrm{~cm}$ of each profile, in which the soil is looser, show higher values, due to agricultural practices or, as in natural profiles, to the annual incorporation of dry vegetal matter. It is important to note that hydraulic conductivity has not increased in the profiles under either reclamation (profile 2) or permanent agricultural use (profile 3) though the salt content has diminished considerably in top layers. This may be due to the swelling and dispersion of clay particles occluding pores responsible for water movement, if their exchangeable Na percentage (ESP), salinity level (table 3) and montmorillonite content (MORENO et al. 1980) are considered. This phenomenon has been discussed by FRENKEL \& RHOADES (1978), amongst others, when studying the influence of dispersion and swelling on hydraulic conductivity of soils. On the other hand, values of this parameter in the top $25 \mathrm{~cm}$ of profile 5 (table 1) are higher than in other cases $(3.2 \mathrm{~cm} / \mathrm{h}$ against about $0.2 \mathrm{~cm} / \mathrm{h}$ ). This could be explained by the lower exchangeable $\mathrm{Na}$ content of this profile (table 3 ) this being a consequence of the reduction of total salt content, in relation to the others, as well as by the relative increase of Ca saturation in the exchange complex preventing the dispersion of clay. All this is undoubtly related to the similar soil use, because of proximity (see fig. 1), of soils in contact with the Marisma, in which zone no drainage and leaching of salts have been carried out. This situation is similar to that described by TANG \& CHANG (1978), in a saline region in China, in which an appropriate agricultural tillage improves hydraulic conductivity in the top horizons.

The coefficient of linear extensibility (COLE) is one of the most clearly differentiating physical parameters (see table 1). Mean values for each profile are, respectively:

$$
8.4-10.5-13.2-8.8-12.6-12.8 \text { (total profile) }
$$

and $7.4-12.0-18.0-9.2-14.5-13.5$ (top $20 \mathrm{~cm}$ )

Profiles 1 and 4 show the lowest COLE, while 3, 5 and 6 show the highest. Profile 3 is subjected to a particular agronomic treatment responsible for the very high value of top layer $(18.0 \%)$. In general, there is an increase for the COLE from natural saline soils to vertisol, with the exception of profile 3 .

Profiles 1 and 4 show a very similar COLE sequence according to depth, with a relative maximum at $30-50 \mathrm{~cm}$. Profile 3 , on the contrary, reaches a minimum at that depth. Differences observed in the COLE, within a profile and between profiles, have to be related to both the use of the soil and the clay fraction mineralogy. In the case of profiles 1 and 4 , the most natural ones, differences observed at various depths are undoubtly due to the montmorillonite content. Although this ranges between 5 and 25\% in soils of the Marisma (MORENO et al. 1980), it is sufficient to produce a large swelling and shrinking effect, due to exchangeable $\mathrm{Na}$ content and to the very high clay fraction percentage (up to 70\%, see table 1). It should be borne in mind that this phenomenon is also affected by the cations adsorbed on the particle surfaces of other clay minerals present in these soils (particularly illite). 


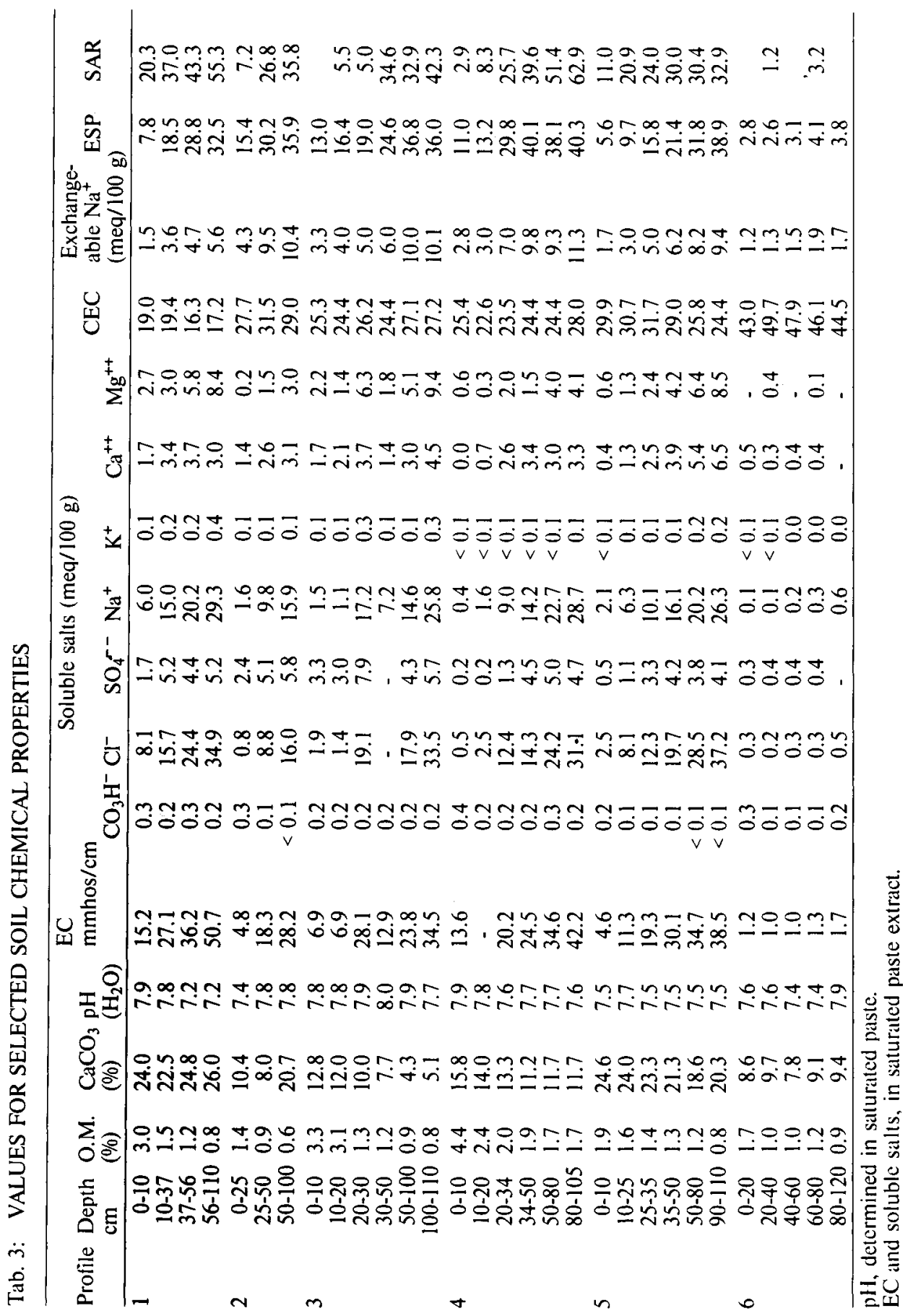



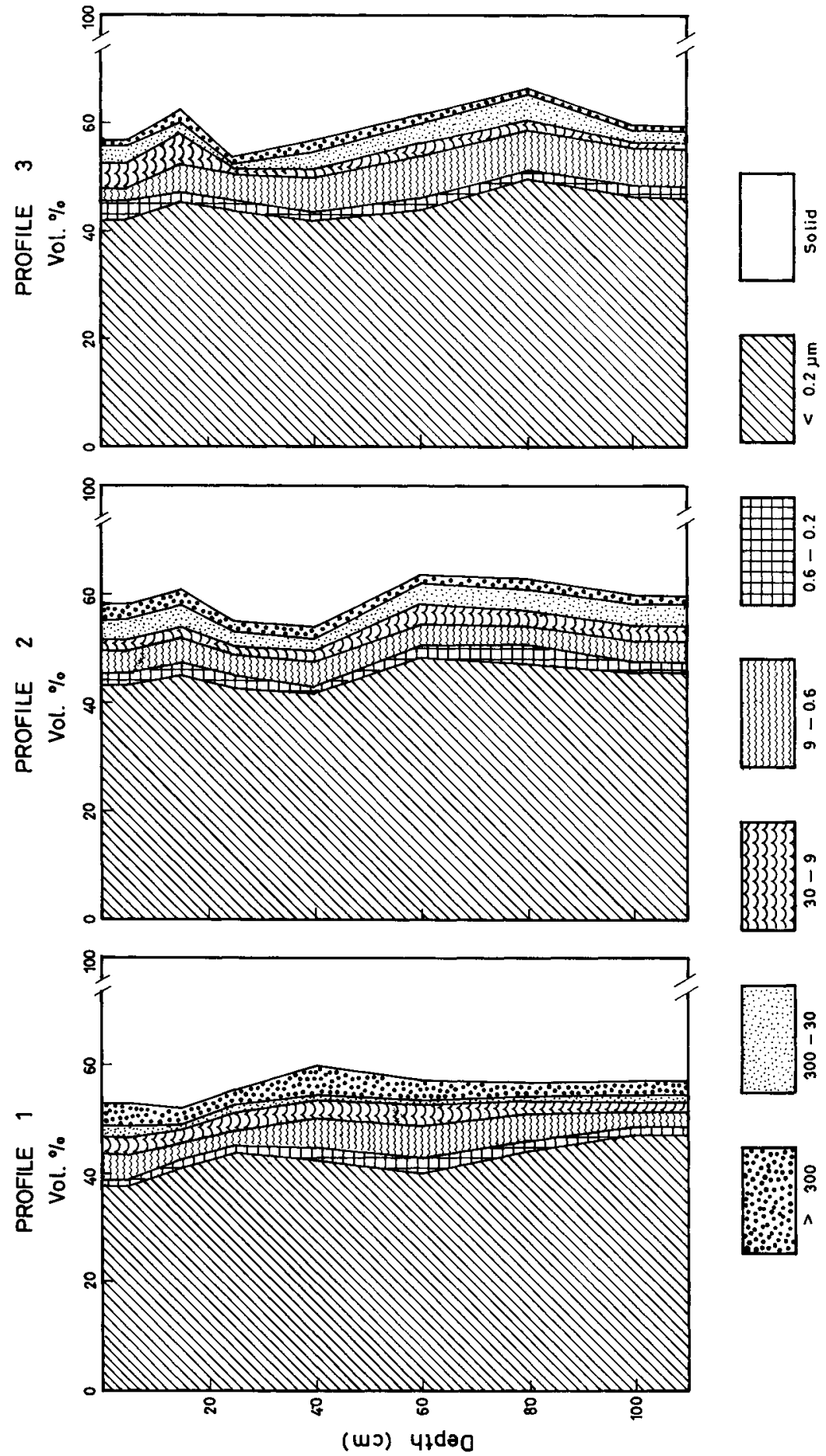

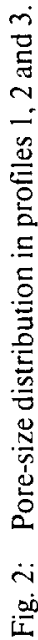



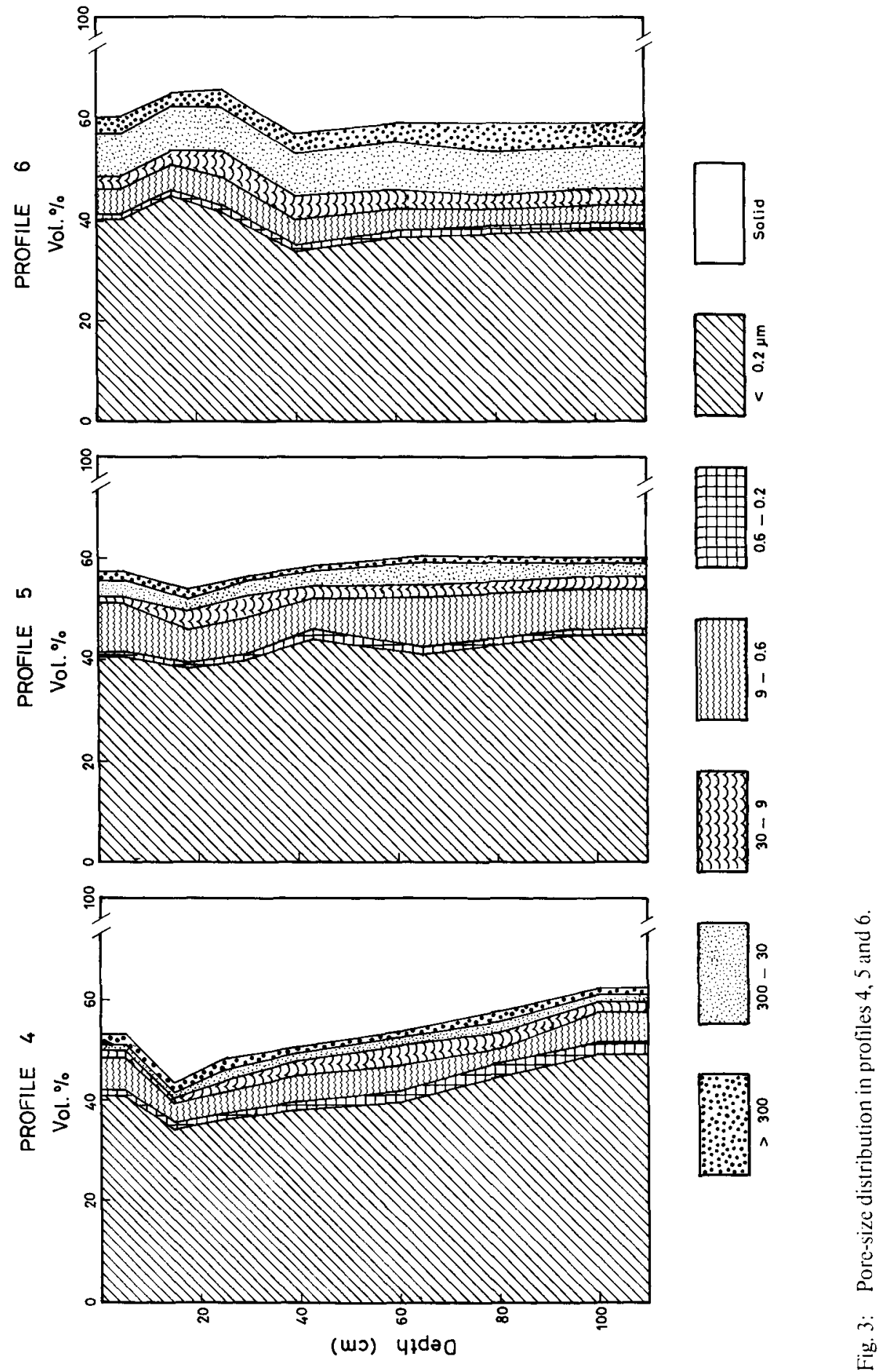
Soils under reclamation schemes or in agricultural use (profile 2, 3 and 5), have still higher values of COLE, and these practices have to be considered as adding their effect to that due to montmorillonite content. The effect is more noticeable in the more desalinized top horizons; in turn this should have contributed to the variation in the ESP of the exchangeable complex. This fact is important because the more the salt content decreases, a little increase of ESP may produce noticeable variations of COLE, as a consequence of the swelling of montmorillonitic material, provided ESP is within the values observed in these soils (table 3). These findings are in agreement with results shown by MACNEAL et al. (1966), when studying the effect of the composition of the solution on the swelling of clays extracted from soils. It should be taken into account that the decrease of soluble salts increases the dispersion of particles, which favours the increase in the COLE. On the other hand, the results are also in agreement with values obtained by ourselves when studying the variation of the COLE due to washing of salts from the samples. The results will be the object of a future paper.

The phenomenon is more obvious in the soils represented by profile 3 (top layers), due to the agricultural practice of incorporating the rice straw when the soil is still water saturated. In the vertisols represented by profile 6 , although influenced by their use, the high montmorillonite content remains as the most important factor responsible for their high COLE.

Another similarity between certain profiles is shown by the fact that correlation between the water holding percentage at $\mathrm{pF} 2.5$ and the COLE rises from $r=0.63$, when considering all depths in all profiles, to $r=0.78$, for only saline soils, excluding the vertisol. When the correlation is done between values for the vertisol and the top three layers of profile 3 and 5 , then $r=0.90$. It should however be noticed that the COLE, as an analytical index, may not be coincident with a vertic character (cf. profile 3 ), when certain unfavourable circumstances are present, as for instance when floading is permanent.

\subsection{PORE-SIZE DISTRIBUTION}

Figs. 2 and 3 present the distribution of pore-size ranges corresponding to each profile. Pore-size distribution shows that the fraction greater than $9 \mu \mathrm{m}$ which is responsible for aeration and drainage, represents a low percentage of the total porosity which is normal considering the high content of fine particles. It is important however to analyze certain clear differences found between profiles. The largest amount of pores greater than $300 \mu \mathrm{m}$ (fast drainage and aeration) is found in profile 6 (fig. 3), the vertisol, in which a very considerable fraction of pores $300-30 \mu \mathrm{m}$ (drainage and aeration) is also present. Taken together with the fraction $30-9 \mu \mathrm{m}$, this represents a well balanced system, that can be explained by the fact that the vertisol in this zone shows a very good structure with very high stability (mean instability index $\left(\mathrm{I}_{\mathrm{S}}\right)$ between 1.5 and 2.0), according to the scale given by ARRUE (1977) for the soils in this region, based on the method of HENIN et al. (1972).

Regarding pores of the fraction 9-0.2 $\mu \mathrm{m}$ it should be noticed that the fraction $9-0.6 \mu \mathrm{m}$, responsible for the storage of easily available water for plants, is predominant in relation to pore fraction $0.6-0.2 \mu \mathrm{m}$ (strongly held available water). These two pore fractions are practically the same in all horizons, which differs from the results of ARRUE (1977) for vertisols with similar characteristics located nearby, who found fewer pores $9-0.2 \mu \mathrm{m}$ in the top layer than in subsoil. Although both these soils show similar structure and stability, this fact could be explained by differences in crop rotations and other agricultural practices producing a looser top layer. 
In the other profiles, representative of saline soils, the fractions of pores greater than $9 \mu \mathrm{m}$ (figs. 2 and 3 ) are less abundant than in the case of the vertisol, which can be partially attributed to the larger number of fine particles. Profiles 2 and 5, which correspond respectively to soils cultivated after drainage and to a naturally well drained cultivated soil, show a good balance of the three pore fractions: greater than $300 \mu \mathrm{m}, 300-30 \mu \mathrm{m}$ and $30-9 \mu \mathrm{m}$. This improvement of structure in the top layers was due to agricultural use. Profile 3 , on the contrary, shows a different balance according to depth, again as a result of its particular use for rice cultivation. A minimum value is found for all fractions between 20 and $40 \mathrm{~cm}$ depth due to the use of mechanical implements.

The natural soils (profiles 1 and 4) have certain differences particularly in the distribution of the fractions greater than $9 \mu \mathrm{m}$. Differences between these two profiles, for drainage and aeration pore fraction, may be due to a different $\mathrm{CaCO}_{3}$ content. This is lower in profile 4 (table 3) and contributes to fewer pores of this size, as the $\mathrm{CaCO}_{3}$ is finely divided (predominant fraction smaller than $2 \mu \mathrm{m}$ ) and it probably acts as a cementing agent between particles. STAKMAN \& BISHAY (1976) have observed that in loamy-clayey soils with different amounts of $\mathrm{CaCO}_{3}$, the aeration porosity diminished on the removal of $\mathrm{CaCO}_{3}$.

In all the saline profiles, pores between 9 and $0.2 \mu \mathrm{m}$ are less abundant than in the vertisol (profile 6), except profile 5 in which the reverse happens. This could be due both to the type of soil use and to more particles in the fraction $2-20 \mu \mathrm{m}$. Another fact is that in profiles 1 , 2,3 and 4 the proportion of pores with size between 0.6 and $0.2 \mu \mathrm{m}$, is slightly higher than in profiles 5 and 6 , the meaning of which will be discussed in the following paragraph.

\subsection{WATER HOLDING CAPACITY}

In all the profiles studied, water holding capacities are high (see table 2 ) as is normal for clayey soils. However, large differences can be observed between profiles: profiles 1 and 4 , as it is the case for the COLE, show the lowest values, while profile 3 shows the highest; this is practically the maximum found in all saline and heavy soils studied in the region.

Water retention at different $\mathrm{pF}$ levels depends on the pore-size distribution shown in figs. 2 and 3. In profiles kept under natural conditions water holding values from $\mathrm{pF} 0$ to pF 4.2 are lower ( $10.5 \%$ in top layers of profile $4,12.9 \%$ in profile 1$)$ than in vertisol $(20.5 \%)$; between $\mathrm{pF} 0$ and 2.5 the values are $4.2,7.8 \mathrm{vs.} 14.6 \%$, respectively. If water holding capacity in profiles 1 and 4 is compared with that in other profiles, clear differences exist, the values being lower in natural profiles, particularly in top layers. This fact could be related to both a different pore-size distribution (figs. 2 and 3 ) and a higher particle dispersion in top layers of profiles 2, 3 and 5 , as a consequence of the lower salt content (table 3 ), previously mentioned. Dispersion is favoured by a larger water accumulation round the cations adsorbed on particle surfaces, thus increasing water holding capacity.

Differences can also be observed between soils in relation to $\mathrm{pF}$ value changes with depth. Water content profiles, at $\mathrm{pF} 2.5$, are given in fig. 4 , so that the differences can be more easily appreciated (see also table 2). The changes under natural conditions (profiles 1,4 ) are not very important and they follow a rather similar pattern. However, in profile 3 a change occurs at $20-50 \mathrm{~cm}$ depth, for the water content is lower. This is in agreement with both the increase of bulk density, observed at the same depth, and the fewer pores greater than $9 \mu \mathrm{m}$ (see table 1 and fig. 2, respectively), and is very probably due to mechanical compaction by machinery. The vertisol shows a quasi homogeneous pattern, except for the top layer in which values are higher. In the top layers of profiles 2 and 5 , which are not represented in 


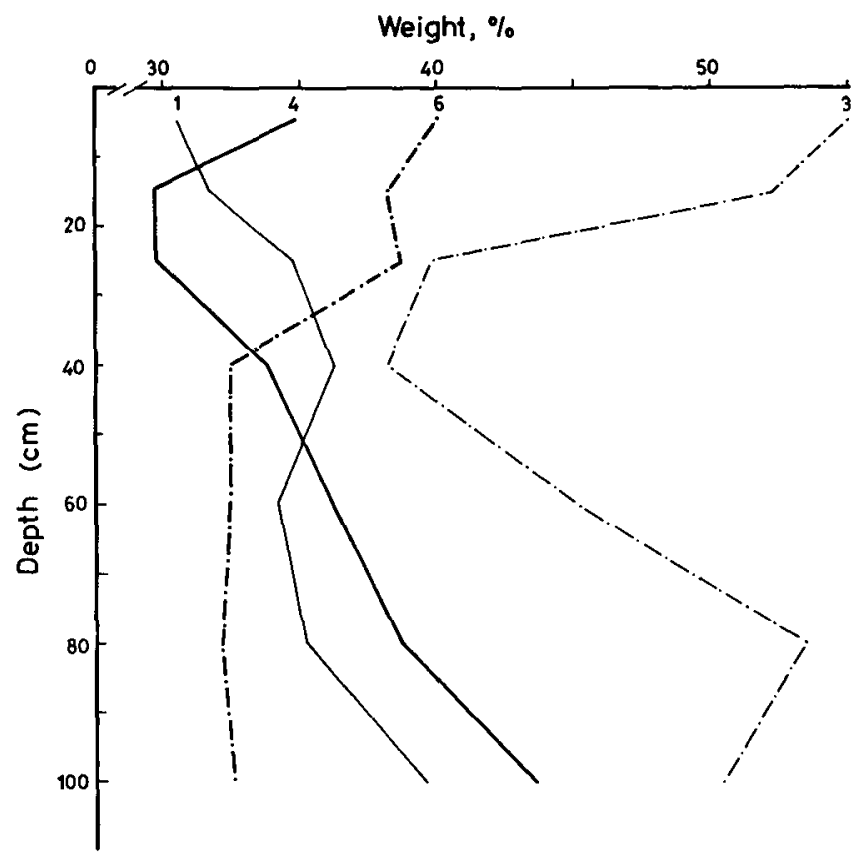

Fig. 4: Water retention at $\mathrm{pF} 2.5$ in given profiles.

fig. 4, the pattern for this and other properties is similar to that of the vertisol. At deeper layers, they are similar to those of the natural saline profiles. The behaviour of top layers is related to soil use.

In profiles $1,2,3$ and 4 , the amount of useful water strongly held represents an important quantity (approx. 40\%) of total useful water, this being in agreement with the porosity data. This means a greater effort by the plants to extract that water.

\subsection{CHEMICAL AND PHYSICO-CHEMICAL PROPERTIES}

Data corresponding to chemical and physico-chemical properties of these soils are included in table 3 and have been determined with the aim of characterizing these soils from the point of view of their salinity and also as an aid in interpreting other properties and phenomena presented in this paper.

The organic matter content is usually low, as it is the rule in cultivated soils under semiarid climatic conditions in the Mediterranean area, except in top layers of profiles 1 and 4, which have been kept under natural conditions.

$\mathrm{CaCO}_{3}$ content, on the contrary, is high, particularly in profiles 1 and 5 . It is finely divided, about half of its quantity (in some cases up to 70\%) being formed by particles smaller than $2 \mu \mathrm{m}$.

$\mathrm{pH}$ corresponds to values for saline, non sodic soils; normally below 8.0 . EC, excluding profile 6 (vertisol) is always high. Data from profile 1 (saline, natural), shown in table 3, are very similar to those found by AYERS et al. (1960) when analyzing samples of soil represen- 
Tab. 4: GYPSUM CONTENT (\%) IN PROFILES 1 AND 4

\begin{tabular}{ccc}
\hline Depth & Profile 1 & Profile 4 \\
\hline $0-10$ & 0.0 & 0.0 \\
$10-20$ & 0.0 & 0.0 \\
$20-30$ & 1.6 & 0.0 \\
$30-50$ & 1.1 & 1.9 \\
$50-70$ & 1.0 & 2.1 \\
$70-90$ & 0.6 & 1.0 \\
$90-110$ & 0.1 & 1.9 \\
\hline
\end{tabular}

Tab. 5: SALT CONTENT OF GROUND WATER IN PROFILE 1

\begin{tabular}{lrl}
\hline $\mathrm{EC}$ & 95.0 & $\mathrm{mmhos} / \mathrm{cm}$ \\
$\mathrm{CO}_{3} \mathrm{H}^{-}$ & 2.2 & meq/l \\
$\mathrm{Cl}^{-}$ & 1420.0 & meq/l \\
$\mathrm{SO}_{4}{ }^{--}$ & 132.8 & $\mathrm{meq} / 1$ \\
$\mathrm{Na}^{+}$ & 1086.9 & $\mathrm{meq} / \mathrm{l}$ \\
$\mathrm{Ca}^{++}$ & 84.8 & $\mathrm{meq} / 1$ \\
$\mathrm{Mg}^{++}$ & 349.5 & $\mathrm{meq} / 1$ \\
\hline
\end{tabular}

ted by this profile. Data corresponding to profile 2 (saline under reclamation and use) are much lower however. This shows the effect of reclamation and subsequent use. Table 4 shows there is no gypsic horizon, even in profile 1 and 4 , in which the gypsum content is highest.

Profile 1, the most recent soil, shows no $\mathrm{CaCO}_{3}$ mobilization; profile 2, far from the river and cultivated, has had much $\mathrm{CaCO}_{3}$ leached. Profile 2 shows no $\mathrm{CaCO}_{3}$ leaching and a certain salt accumulation at $20-30 \mathrm{~cm}$.

In general, Na-saturation is greater than 15\%. Table 5 includes values for salt content in ground water. $\mathrm{Cl}^{-}, \mathrm{Na}^{+}$and $\mathrm{Mg}^{++}$are the main ions present.

Although it is not included in the tables, the clay mineral fraction is predominantly formed by illite, in an advanced degree of alteration. Small quantities of montmorillonite and kaolinite are present. Traces of chlorite are also found. Usually montmorillonite never accounts for more than $10 \%$ of the total mineral fraction though exceptionally it might reach $25 \%$ in certain horizons (MORENO et al. 1980).

\section{CONCLUSIONS}

The soils studied show differences due to situation, parent material and use. All of them, however, are recent soils of fine texture.

All these soils show high values of microporosity: $0.7-0.8$ of total porosity correspond to the pore size range smaller than $0.2 \mu \mathrm{m}$. On the other hand, fractions of pores greater than 9 $\mu \mathrm{m}$ keep a good balance in profile 6 (vertisol), 2 and 5 (saline cultivated soils), although in the latter, this pore-size fraction is less abundant.

Hydraulic conductivity is very low and only in the top layers of profiles of pronounced vertic character are higher values observed, as in profile 5.

Water holding capacity is always high as is normal in clayey soils. Of greater importance is the high proportion of strongly held useful water (pores between 0.6 and $0.2 \mu \mathrm{m}$ ), in saline soils.

The COLE, in general, increases from soils under natural conditions to vertisols. According to this and morphological observations, the vertic characteristics increase progressively from those areas near the river to the marginal zones in contact with vertisols.

From previous data it could be deduced that the existing relationship between vertisol and saline soils is due to the vertic character of the latter soils and, as a consequence, to the derived properties. Although this vertic character could be produced by reclamation and use of the soil, it should be accepted that further research is needed to find out what transfor- 
mations occur in clay minerals when the soil is desalinized. We have not found, in the available references, works dealing with the relationships between saline soils and vertisols.

These soils have been classified by GIRALDEZ \& CRUZ (1975) as saline-alkaline soils, according to the criteria of the U.S. Salinity Laboratory. We propose, from the results obtained in the present work and according to the U.S.D.A. (1975), the following classification for the soils:

Profile 1 , the most recent one, shows a slightly developed structure. In view of its physical and physico-chemical properties, Na-saturation, EC and pH values, it should be considered as an alkali-saline soil, according to the Soil Salinity Laboratory classification. Following the U.S.D.A it should be classified as Fluvaquent (saline phase).

Profile 2 has a more developed structure. Although still an alkali-saline soil, it could be altered into a non-saline alkali through the leaching of salts. It is a Vertic Haplaquet, with salt content increasing with depth.

Profile 3 has a slightly developed structure, due to permanent flooding, with no vertic characteristics; therefore it should be classified as Typic Haplaquept, with salt content increasing with depth.

Profile 4 shows a strong vertic character. As with profile 1 it is less expandible than the others. Na-saturation is smaller than $15 \%$, in the first $20 \mathrm{~cm}$, and increases with depth and the soil is saline (EC is greater than 4 mmhos; SAR is greater than 13 from $20 \mathrm{~cm}$ downwards). From these data, the soil should be considered as Entic Chromoxerert.

Profile 5 also has a strong vertic character, and with the COLE greater than 14\%, it is an alkali-saline soil, with $\mathrm{CaCO}_{3}$ content higher in the top $50 \mathrm{~cm}$, as a consequence of the nearby marly-calcareous areas. It is an intergrade Entic Pelloxerert to Vertic Haplaquept.

Finally, profile 6 is a vertisol. Considering physical and chemical data (and colour "value"), it should be classified as Entic Pelloxerert.

\section{ACKNOWLEDGEMENTS}

Thanks are due to Dr. J.M. Murillo for his contribution in describing vegetation, and to Tech. Ing. M. Ruiz for help with laboratory analyses.

\section{BIBLIOGRAPHY}

ARRUE, J.L. (1977): Factores quimicos, fisico-quimicos y fisicos determinantes de los caracteres, propiedades y dinámica de la porosidad de los suelos. Doctoral Thesis. University of Seville. Spain.

AYERS, A.D., VAZQUEZ, A, DE LA RUBIA, J., BLASCO, F. \& SAMPLON, S. (1960): Saline and sodic soils of Spain. Soil Sci. 90(2), 133-138.

DE LEENHEER, L., VAN RUYMBEKE, M. \& MAES, L. (1965): The chain-hydrometer method for particle size analysis. Z. Pflanzenernähr. Düng. Bodenk. 68, 10-19.

FLANNERY, RD. \& KIRKHAM, D. (1964): A soil core water permeameter for field use. Soil Sci. 97, 233-241.

FRENKEL, H. \& RHOADES, J.D. (1978): Effects of dispersion and swelling on soil hydraulic conductivity. Journal of Testing and Evaluation 6(1), 60-65.

GIRALDEZ, J.V. \& CRUZ ROMERO, G. (1975): Salt movement in Guadalquivir marshy soils under field and laboratory conditions. Egypt. J. Soil Sci. 15(1), 79-93.

GONZALEZ GARCIA, F., GONZALEZ GARCIA, S. \& CHAVES SANCHEZ, M. (1956): The alkali soils of the lower valley of the Guadalquivir: physico-chemical properties and nature of their clay fraction. Proc. Vth Intern. Congress Soil Sci. B(I.26), 185-191.

GRANDE COVIAN, R. (1967): Las marismas del Guadalquivir y su rescate. Ministerio de Agricultura, vol. 5 No. 29. Madrid (Spain). 
GRANDE COVIAN, R (1976): Experiencias en España con el drenaje subterráneo. Estudios sobre riego y avenamiento. El drenaje de suelos salinos. FAO, Roma.

HENIN, S., GRAS, R \& MONNIER, G. (1972): El perfil cultural. El estado fisico del suelo y sus consecuencias agronómicas. Ed. Mundi-Prensa. Madrid.

HIDALGO, L. \& CANDELA, M.R. (1958): El poder clorosante de los suelos calizos en el viñedo. Boln. Inst. Nac. Invest. Agron. 18,383 (Madrid).

KYUMA, K. \& KAWAGUCHI, K (1967): The classification of soils under rice cultivation (Paddy soils): Anal. Edafol. Agrobiol. 26, 439-446.

LEYVA CABELLO, F. (1976): Memoria explicativa de la hoja geológica 1:50000, No. 1018, "El Rocio". I.G.M.E. Madrid.

MARTIN ARANDA, J. (1973): Factores fisicos fundamentales en la economia de agua de los suelos de Andalucia Occidental. Alcance agronómico en cultivos de regadio. Doctoral Thesis. University of Granada. Spain.

MARTIN, J., MORENO, F. \& ARRUE, J.L. (1978): Clay minerals and chemical fertility improvement in some representative SW. Spain soils. Proc. Intern. Symposium "Soil fertility improvement and clay minerals" II, 262-270 (Prague, Czechoslovakia).

McNEAL, B.L., NORVELL, W.A. \& COLEMAN, N.T. (1966): Effect of solution composition on the swelling of extracted soil clays. Soil Sci. Soc. Amer. Proc. 30, 313-317.

MORENO, F., ARRUE, J.L., MURILLO, J.M., PEREZ RODRIGUEZ, J.L. \& MARTIN, J. (1980): Mineralogical composition of clay fraction in marsh soils of SW. Spain. Polish J. Soil Sci. (in press).

MORENO, F. \& MARTIN, J. (1979): Influence of the tillage system on physical properties in some SW. Spain soils. Proc. 8th Conference of the International Soil Tillage Research Organization. I, 57-62 (Stuttgart).

MORENO, F., MARTIN, J. \& ARRUE, J.L. (1978): Clay minerals and physical fertility improvement in some representative SW. Spain soils. Proc. Intern. Symposium "Soil fertility improvement and clay minerals" II, 271-280 (Prague, Czechoslovakia).

PIPER, S.C. (1950): The determination of organic carbon: Walkley and Black's rapid titration method. In: Soil and Plant analysis, 223-227, University of Adelaide.

RJCHARDS, L.A (1948): Porous plate apparatus for measuring moisture retention and transmission by soil. Soil Sci. 66, 105-110.

RICHARDS, L.A (1954): Diagnosis and improvement of saline and alkali soils. U.S. Dept. Agri. Handb. No. 60 .

U.S.D.A. (1975): Soil Taxonomy: A basic system of soil classification for making and interpreting soil surveys. U.S.D.A. Agri. Handb. 436, U.S. Govm. Printing Office, Washington D.C.

STAKMAN, W.P. \& BISHAY, B.G. (1976); Moisture retention and plasticity of highly calcareous soils in Egypt. Neth. J. Agric. Sci. 24, $43-57$.

TANG, S.Y. \& CHANG, L.C. (1978): Effect of the development of the ploughed horizon on the movement of soil water and soluble salts in saline soils of the coastal area northern Kiangsu. Acta Pedologica Sinica 15(1), 39-52.

VOMOCIL, J.A (1965): Porosity. (In: C.A. Black ed, Methods of soil analysis. Part I. Agronomy 9, 299. 314. Amer. Soc. Agron., Madison, Wisconsin). 